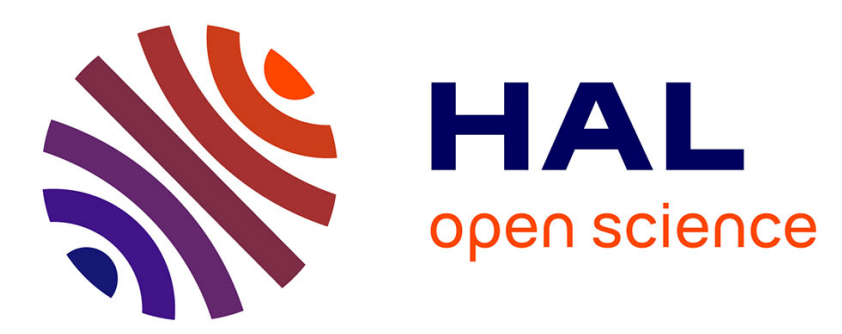

\title{
Plasmon dispersion of the strongly coupled one component plasma in two and three dimensions
}

\author{
J.P. Hansen
}

\section{To cite this version:}

J.P. Hansen. Plasmon dispersion of the strongly coupled one component plasma in two and three dimensions. Journal de Physique Lettres, 1981, 42 (17), pp.397-400. 10.1051/jphyslet:019810042017039700 . jpa-00231956

\section{HAL Id: jpa-00231956 https://hal.science/jpa-00231956}

Submitted on 1 Jan 1981

HAL is a multi-disciplinary open access archive for the deposit and dissemination of scientific research documents, whether they are published or not. The documents may come from teaching and research institutions in France or abroad, or from public or private research centers.
L'archive ouverte pluridisciplinaire HAL, est destinée au dépôt et à la diffusion de documents scientifiques de niveau recherche, publiés ou non, émanant des établissements d'enseignement et de recherche français ou étrangers, des laboratoires publics ou privés. 


\title{
Plasmon dispersion of the strongly coupled one component plasma in two and three dimensions
}

\author{
J. P. Hansen \\ Institut Laue-Langevin, 156X, 38042 Grenoble Cedex, France \\ (Reçu le 1 ${ }^{\text {er }}$ juin 1981, accepté le 8 juillet 1981)
}

\begin{abstract}
Résumé. - On montre que la dispersion négative et le maximum du taux d'amortissement des ondes électrostatiques sont des caractéristiques communes aux plasmas fortement corrélés à deux et à trois dimensions.
\end{abstract}

Abstract. - We show that negative plasmon dispersion and a damping rate maximum are common features of highly correlated plasmas in two and three dimensions.

In this letter we compare the influence of strong correlations on plasmon dispersion and damping in two and three space dimensions. For this purpose we consider the simple model of a one component plasma (OCP) made up of point charges $e$ immersed in a uniform neutralizing background. The Coulomb potential is $v(r)=e^{2} / r$ in three dimensions (3d) and $-e^{2} \ln (r / L)$ (with $L$ an arbitrary scale factor) in two dimensions (2d); a convenient unit of length is the ion sphere (disk) radius $a=(3 / 4 \pi n)^{1 / 3}\left((1 / \pi n)^{1 / 2}\right)$, where $n$ is the number density, and the usual dimensionless coupling constant is $\Gamma=\beta e^{2} / a\left(\beta e^{2}\right)$, where $\beta=1 / k_{\mathrm{B}} T$ is the inverse temperature. In $3 \mathrm{~d}$ extensive computer simulations [1] and a number of theoretical studies $[2,3]$ have shown that for coupling constants $\Gamma \gtrsim 1$, plasmon dispersion and damping differs markedly from mean field behaviour; in particular the dispersion $\mathrm{d} \omega / \mathrm{d} k$ becomes negative for $\Gamma \gtrsim 10$. The $2 \mathrm{~d}$ case is of interest because " particle " simulations of inertially confined fusion plasmas are generally restricted to $2 \mathrm{~d}$ for practical reasons, and also because detailed information on static properties of the $2 \mathrm{~d}$ OCP has recently become available $[4,5]$.

We recall that, if frequencies are scaled by the plasma frequency $\omega_{\mathrm{p}}$ and wave-numbers by the Debye wave-number $k_{\mathrm{D}}$, the dielectric function $\varepsilon(k, \omega)$ is independent of dimensionality in the mean field (i.e. collisionless Vlasov) approximation. The main purpose of this work is to investigate the influence of dimensionality on plasma waves in the presence of strong particle correlations.

The basic dynamical variable in our problem is the microscopic longitudinal current density, which has Fourier-components :

$$
j_{\mathbf{k}}^{1}(t)=\frac{1}{v_{0} k} \sum_{i=1}^{N} \mathbf{k} \cdot \mathbf{v}_{i}(t) \mathrm{e}^{-i \mathbf{k} \cdot \mathbf{r}_{i}(t)}=\frac{i}{v_{0} k} \frac{\mathrm{d} \rho_{\mathbf{k}}(t)}{\mathrm{d} t}
$$

where $v_{0}=\left(k_{\mathrm{B}} T / m\right)^{1 / 2}$ is the thermal velocity, $\mathbf{r}_{i}(t)$ and $\mathbf{v}_{i}(t)$ denote the position and velocity of particle $i$ at time $t$, and $\rho_{\mathbf{k}}(t)$ is the microscopic density. The Laplace transform (denoted by a tilda) of the longitudinal current correlation function :

$$
C_{1}(k, t)=\frac{1}{N}\left\langle j_{\mathbf{k}}^{1}(t) j_{\mathbf{k}}^{1 *}(0)\right\rangle
$$

satisfies the generalized Langevin equation [6] :

$$
\left[-i \omega+\frac{i}{\omega} \frac{v_{0}^{2} k^{2}}{S(k)}+\tilde{M}_{1}(k, \omega)\right] \tilde{C}_{1}(k, \omega)=1
$$

where $S(k)=\left\langle\rho_{\mathbf{k}} \rho_{-\mathbf{k}}\right\rangle / N$ is the static structure factor and $\tilde{M}_{1}(k, \omega)$ is the memory function.

If $\tilde{C}_{1}(k, \omega)$ is known, the fluctuation-dissipation theorem yields directly the dielectric function :

$$
\varepsilon^{-1}(k, \omega)=1-i \frac{\omega_{\mathrm{p}}^{2}}{\omega^{2}} \tilde{C}_{1}(k, \omega)
$$

We now relate $\tilde{C}_{1}(k, \omega)$ to the corresponding correlation function, $\widetilde{C}_{1}^{(0)}(k, \omega)$, of some reference motion, by writing down the generalized Langevin equation 
for $\tilde{C}_{1}^{(0)}(k, \omega)$, and subtracting it from equation (3), with the result :

$$
\begin{aligned}
& \left\{\left[\tilde{C}_{1}^{(0)}(k, \omega)\right]^{-1}-\frac{i}{\omega} v_{0}^{2} k^{2} \Delta \hat{c}(k)+\right. \\
& \left.+\Delta \tilde{M}_{1}(k, \omega)\right\} \tilde{C}_{1}(k, \omega)=1
\end{aligned}
$$

where

$$
\Delta \hat{c}(k)=\hat{c}(k)-\hat{c}^{(0)}(k)=\left[S^{(0)}(k)\right]^{-1}-[S(k)]^{-1}
$$

is the difference of direct correlation functions and $\Delta \tilde{M}_{1}(k, \omega)$ is the difference of memory functions. One frequently chooses the self motion as the reference dynamics [7], but here we shall make the even simpler choice of extracting the free particle motion [8]. The corresponding $\widetilde{C}_{1}^{(0)}$ reads :

$$
\widetilde{C}_{1}^{(0)}(k, \omega)=-\frac{i \omega}{v_{0}^{2} k^{2}} W\left(\frac{\omega}{v_{0} k}\right)
$$

where $W(x)$ denotes the standard plasma dispersion function :

$$
\begin{aligned}
W(x) & =W^{\prime}(x)+i W^{\prime \prime}(x)= \\
& =1-x \mathrm{e}^{-x^{2} / 2} \int_{0}^{x} \mathrm{e}^{s^{2} / 2} \mathrm{~d} s+i \sqrt{\frac{\pi}{2}} x \mathrm{e}^{-x^{2} / 2}
\end{aligned}
$$

while $\hat{c}^{(0)}(k)=0$. Note that the mean field (Vlasov) form for $\tilde{C}_{1}(k, \omega)$ is then recovered from equation (5) if $\hat{c}(k)$ is replaced by its weak coupling (DebyeHückel) limit $-k_{\mathrm{D}}^{2} / k^{2}$, and if $\Delta \tilde{M}_{1}(k, \omega)$ is neglected. To account for strong correlations, we shall use the exact $\hat{c}(k)$, and make a simple gaussian ansatz for the memory function :

$$
\Delta M_{1}(k, t)=\Omega_{0}^{2}(k) \exp \left\{-t^{2} / 2 \tau^{2}(k)\right\}
$$

where $\tau(k)$ denotes a wave-number dependent relaxation time which characterizes all collisional processes in the plasma. We determine $\Omega_{0}(k)$ and $\tau(k)$ from the high frequency expansion :

$$
\begin{aligned}
& \tilde{C}_{1}(k, \omega)=\frac{i}{\omega} \times \\
& \times\left[1+\frac{\omega_{11}^{(0) 2}(k)+\Delta \omega_{11}^{2}(k)}{\omega^{2}}+\frac{\omega_{21}^{(0) 4}(k)+\Delta \omega_{21}^{4}(k)}{\omega^{4}}\right. \\
& \left.+\mathrm{O}\left(\frac{1}{\omega^{6}}\right)\right]
\end{aligned}
$$

where we have extracted the contribution of the free particle motion to the frequency moments [6] $\omega_{n 1}^{2 n}(k)=\omega_{n 1}^{(0) 2 n}(k)+\Delta \omega_{n 1}^{2 n}(k)$. Combining equations (5), (6), (8) and (9) we obtain :

$$
\Omega_{0}^{2}(k)=\Delta \omega_{11}^{2}(k)+v_{0}^{2} k^{2} \Delta \hat{c}(k)
$$

$$
\tau^{2}(k)=\frac{\Omega_{0}^{2}(k)}{\Delta \omega_{21}^{4}(k)-\Delta \omega_{11}^{2}(k)\left[\Delta \omega_{11}^{2}(k)+2 \omega_{11}^{(0) 2}(k)\right]} .
$$

Expressions for $\Delta \omega_{11}^{2}(k)$ and $\Delta \omega_{21}^{4}(k)$ in terms of the pair and triplet distribution functions are available in the literature for the $3 \mathrm{~d}$ OCP $[9,10]$. For the $2 \mathrm{~d}$ OCP we find :

$$
\Delta \omega_{11}^{2}(k)=\omega_{\mathrm{p}}^{2}\left[1+G_{2}(k)\right]
$$

$$
\begin{aligned}
\Delta \omega_{21}^{4}(k)=\omega_{\mathrm{p}}^{4} & \left\{1+9 \frac{k^{2}}{k_{\mathrm{D}}^{2}}+2 G_{2}(k)+2\left[G_{2}(k)\right]^{2}+\right. \\
+ & \left.12 \frac{k^{2}}{k_{\mathrm{D}}^{2}} G_{3}(k)+K(k)+I(k)\right\} \quad(11 b)
\end{aligned}
$$

with

$$
\begin{gathered}
G_{n}(k)=\int_{0}^{\infty}[g(r)-1] \frac{J_{n}(k r)}{(k r)^{n-2}} \frac{\mathrm{d} r}{r^{3}} \\
K(k)=a^{2} \int_{0}^{\infty} g(r)\left[1-J_{0}(k r)\right] \frac{\mathrm{d} r}{r^{3}} \\
I(k)=\frac{1}{(2 \pi)^{2}} \int \frac{\mathrm{d}^{2} r}{r^{2}} \int \frac{\mathrm{d}^{2} r^{\prime}}{r^{\prime 2}} \cos (2 \alpha) \times \\
\times\left[g^{(3)}\left(r, r^{\prime}, \alpha\right)-g(r) g\left(r^{\prime}\right)\right] \\
\times\left[1-\mathrm{e}^{i \mathbf{k} . \mathbf{r}}-\mathrm{e}^{-i \mathbf{k} \cdot \mathbf{r}^{\prime}}+\mathrm{e}^{i \mathbf{k} .\left(\mathbf{r}-\mathbf{r}^{\prime}\right)}\right] .
\end{gathered}
$$

Here $J_{n}$ is the $n$th order cylindrical Bessel function, $g(r)$ denotes the pair and $g^{(3)}$ the triplet distribution function, with $\alpha$ the angle between the vectors $\mathbf{r}$ and $\mathbf{r}^{\prime}$. To calculate $I(k)$ we must resort to the standard superposition approximation for $g^{(3)}$ [6], except for the case $\Gamma=2$, where $g^{(3)}$ is known exactly [4]. For the pair distribution functions of the $2 \mathrm{~d}$ OCP we have used recent hypernetted chain (HNC) results [5].

Our expressions for $\widetilde{C}_{1}(k, \omega)$ and $\varepsilon(k, \omega)$ are independent of dimensionality. The differences between two and three dimensions for a given coupling $\Gamma$ can hence only stem from differences in the static information contained in $S(k)$ and in the frequency moments $\omega_{n 1}^{2 n}(k)$. The difference between the $2 \mathrm{~d}$ and $3 \mathrm{~d}$ cases appears most clearly in the $k$-dependence of the relaxation time $(10 b)$. In $3 \mathrm{~d}, \omega_{11}^{2}$ and $\omega_{21}^{4}$ behave as $k^{2}$ for small $k[9,10]$ and hence $\tau(k)$ goes to a nonzero constant (say $\tau_{0}$ ) as $k \rightarrow 0$; from the results of $[9,10]$ we conclude that $\omega_{\mathrm{p}} \tau_{0} \simeq 0.23 \Gamma^{1 / 2}$ for strong coupling $(\Gamma>1)$.

In $2 \mathrm{~d}$ the situation is more complicated, since the integrals $K(k)$ and $I(k)$ in equations (12), although well defined for all values of $k$, lack the simple $k^{2}$ behaviour of their 3d counterparts and of the $G_{n}(k)$. In figure 1 we show some numerical results for $\tau$ as a function of $k: \tau(k)$ is seen to rise sharply from zero for small $k$ and to reach a $\Gamma$-dependent plateau value $\tau_{0}$ for $k \leqslant 0.5 / a ;$ numerically $\omega_{\mathrm{p}} \tau_{0} \simeq 0.20 \Gamma^{2 / 5}$. Note that the vanishing of $\tau(k)$ as $k \rightarrow 0$ may well be 


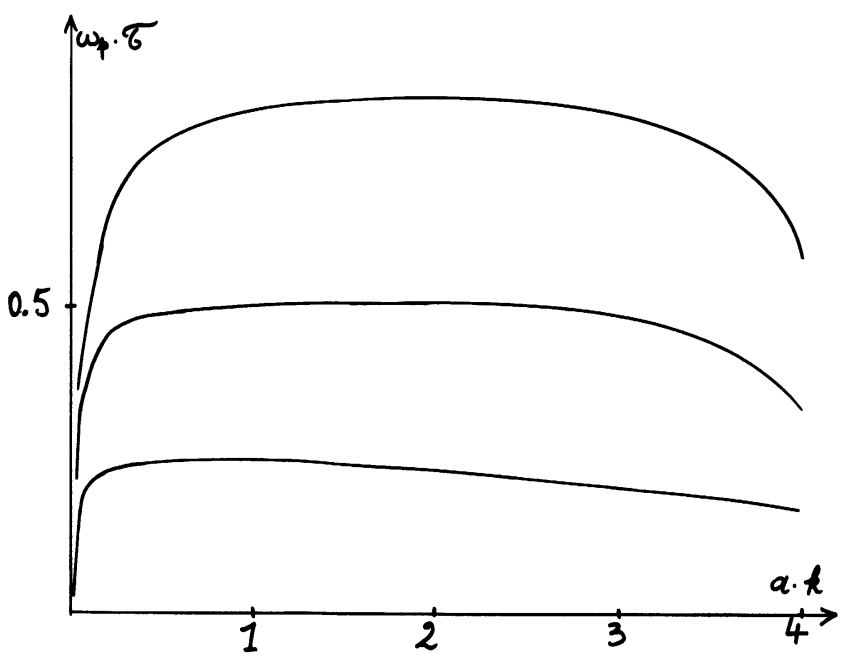

Fig. 1. - Variation of $\omega_{\mathrm{p}} \tau$ (eq. (10b)) with the reduced wavenumber $a . k$ in the $2 \mathrm{~d}$ OCP, for $\Gamma=2,10$ and 40 .

spurious and due to our determination of the relaxation time from a high frequency expansion.

For the sake of simplicity we shall henceforth neglect the $k$-dependence of $\tau$, and replace the relaxation time by the above-mentioned values of $\tau_{0}$ in 2 and 3 dimensions. This approximation is reasonable, as long as we restrict ourselves to $k \lesssim 1 / a$. More specifically, we investigate plasmon dispersion and damping by solving the dispersion relation $\varepsilon(k, \omega)=0$ to order $k^{2}$. Combining equations (4-6), (8), (10) and (11), the dispersion relation is cast in the form :

$$
\begin{aligned}
1+\frac{k_{\mathrm{D}}^{2}}{k^{2}} W\left(\frac{\omega}{v_{0} k}\right)\left\{1+G_{2}(k)-W\left(\omega \tau_{0}\right) \times\right. \\
\left.\times\left[1+G_{2}(k)+\frac{k^{2}}{k_{\mathrm{D}}^{2}} \hat{c}(k)\right]\right\}=0
\end{aligned}
$$

since we restrict ourselves to $k \lesssim 1 / a \ll k_{\mathrm{D}}=\sqrt{\Gamma d} / a$ in the strong coupling limit, we can completely neglect Landau damping (i.e. $W^{\prime \prime}\left(\omega / v_{0} k\right)$ but not $W^{\prime \prime}\left(\omega \tau_{0}\right)$ !). Also, to order $k^{2}$ [2] :

$$
\begin{gathered}
\frac{k^{2}}{k_{\mathrm{D}}^{2}} \hat{c}(k)=-1+\alpha_{1} \frac{k^{2}}{k_{\mathrm{D}}^{2}} \\
\alpha_{1}=1-\left(\frac{\partial \beta P}{\partial n}\right)_{T}=\left\{\begin{array}{l}
\Gamma / 4 \\
\simeq 4 \Gamma / 10 \\
G_{2}(k)=\alpha_{2} \frac{k^{2}}{k_{\mathrm{D}}^{2}}
\end{array}\right. \\
\alpha_{2}=\left\{\begin{array}{l}
-\Gamma / 8 \\
4 u(\Gamma) / 15 \simeq-6 \Gamma / 25
\end{array}\right.
\end{gathered}
$$

where $u=\beta U^{\text {ex }} / N$ denotes the excess internal energy per particle and $P$ the pressure. The $2 \mathrm{~d}$ values quoted for $\alpha_{1}$ and $\alpha_{2}$ are exact, while for $3 \mathrm{~d}$ we quote the "ion-sphere » values, which are valid for $\Gamma \gg 1$ [2].
Combining now equations (13)-(15), we obtain the desired dispersion and damping to order $k^{2}$ :

$$
\begin{aligned}
\frac{\omega(k)}{\omega_{\mathrm{p}}} & =1+\frac{3}{2} \frac{k^{2}}{k_{\mathrm{D}}^{2}}\left[1+\frac{\delta_{1}}{3}\right]+i \frac{\delta_{2}}{2} \frac{k^{2}}{k_{\mathrm{D}}^{2}} \\
\delta_{1} & =\alpha_{2}-\left(\alpha_{1}+\alpha_{2}\right) W^{\prime}\left(\omega_{\mathrm{p}} \tau_{0}\right) \\
\delta_{2} & =-\left(\alpha_{1}+\alpha_{2}\right) W^{\prime \prime}\left(\omega_{\mathrm{p}} \tau_{0}\right) .
\end{aligned}
$$

Since $1 \geqslant W^{\prime}(x)>-0.285 \ldots$ and $W^{\prime \prime}(x)>0$, it is clear from equations (14)-(15) that $\delta_{1}$ and $\delta_{2}$ are always negative. Moreover $\left|\delta_{1}\right|$ increases with $\Gamma$, so that we expect negative dispersion beyond some critical $\Gamma_{\mathrm{c}}$. In $3 \mathrm{~d}$ we find $\Gamma_{\mathrm{c}} \simeq 10$, in good agreement with simulation data [1] and other theoretical estimates [2, 3], while in $2 \mathrm{~d}, \Gamma_{\mathrm{c}} \simeq 14$.

For a fixed value of the reduced wave-number $a . k$, collisional damping is characterized by $\Delta_{2}(\Gamma)=\delta_{2} / a^{2} k_{\mathrm{D}}^{2}=\delta_{2} / d . \Gamma$; combining equations (7), (14)-(16), and the previous expressions for $\tau_{0}$, we find :

$$
\Delta_{2}(\Gamma)=-c \Gamma^{\gamma} \mathrm{e}^{-a \Gamma^{2 \gamma}}
$$

where $c \simeq 0.015(0.015), a \simeq 0.020 \quad(0.025)$ and $\gamma=0.4(0.5)$ in 2 (3) dimensions. As expected collisional damping vanishes in the weak coupling limit [11]. But equation (17) also shows that the damping rate is maximum for $\Gamma \simeq 56(20)$ in $2 \mathrm{~d}(3 \mathrm{~d})$ and decreases for stronger couplings, a behaviour which is compatible with available simulation data in $3 d$ [1].

Although our simple calculation has no pretention of rigour, its predictions should be qualitatively, and even semi-quantitatively correct; as illustrated by the good agreement with the computer results in $3 \mathrm{~d}$. Whilst an exact correspondence between two and three-dimensional plasmon propagation holds only in the collisionless regime, the similarities remain very striking even in the presence of strong correlations. This means that the simulation of collective behaviour in real $3 \mathrm{~d}$ dense plasmas by $2 \mathrm{~d}$ codes should be reliable, even in the strong coupling regime.

It is however worth pointing out that significant differences between the $2 \mathrm{~d}$ and $3 \mathrm{~d}$ OCP show up in the high frequency behaviour of the single particle (self) motion; this motion is characterized by the normalized velocity autocorrelation function $Z(t)$ which is the $k \rightarrow 0$ limit of the self part, $C_{1}^{(\mathrm{s})}(k, t)$, of the longitudinal current correlation function (2). Extracting the self contributions to the frequency moments in equations (9), (11) and (12), we find for the $2 \mathrm{~d}$ OCP :

$$
\omega_{11}^{(\mathrm{s})} 2(k)=\frac{\omega_{\mathrm{p}}^{2}}{2}+3 v_{0}^{2} k^{2}
$$

$$
\begin{aligned}
\omega_{21}^{(\mathrm{s}) 4}(k)=\omega_{\mathrm{p}}^{4}\left[\frac{1}{4}+\right. & \left.K^{(\mathrm{s})}+I^{(\mathrm{s})}\right]+ \\
& +\frac{15}{2} \omega_{\mathrm{p}}^{2} v_{0}^{2} k^{2}+15 v_{0}^{4} k^{4}
\end{aligned}
$$


where $K^{(s)}$ and $I^{(\mathrm{s})}$ are the $k$-independent parts of the integrals $K(k)$ and $I(k)$ in equations (12); in particular :

$$
K^{(\mathrm{s})}=a^{2} \int_{0}^{\infty} g(r) \frac{\mathrm{d} r}{r^{3}} .
$$

Since $g(r) \sim \exp \{-\beta v(r)\} \sim r^{\Gamma}$ for $r \rightarrow 0$, it is immediately clear that the integral $(18 c)$ diverges for $\Gamma \leqslant 2$; no such singularity occurs for the $3 \mathrm{~d}$ case.
We conclude that the fourth frequency moment of the spectral function associated with $C_{1}^{\mathrm{s})}(k, t)$, and hence with $Z(t)$, diverges for $\Gamma \leqslant 2$; in other words, for sufficiently high temperatures, these spectral functions exhibit a high frequency tail which decays more slowly than $1 / \omega^{5}$ in $2 \mathrm{~d}$, while the corresponding $3 \mathrm{~d}$ spectral functions are expected to decay exponentially. This high frequency tail might be a consequence of the extreme «softness " of the Coulomb potential in two dimensions.

\section{References}

[1] Hansen, J. P., McDonald, I. R. and Pollock, E. L., Phys. Rev. A 11 (1975) 1025.

[2] For a review, see Baus, M. and Hansen, J. P., Phys. Rep. 59 (1980) 1.

[3] Carini, P., Kalman, G. and Golden, K. I., Phys. Lett. 78A (1980) 450.

[4] Alastuey, A. and Jancovici, B., J. Phy.ique 42 (1981) 1. JanCOvici, B., Phys. Rev. Lett. 46 (1981) 386.

[5] Bakshi, P., Calinon, R., Golden, K. I., Kalman, G. and Merlini, D., Phys. Rev. A 23 (1981) 1915.

HANSEN, J. P. and LeVesque, D., to be published.
[6] See e.g. Hansen, J. P. and McDonald, I. R., Theory of simple Liquids, (Academic Press, London) 1976.

[7] Suögren, L., J. Phys. C 13 (1980) L841.

[8] Takeno, S. and Yoshida, F., Prog. Theor. Phys. 62 (1979) 883.

[9] Vieillefosse, P. and Hansen, J. P., Phys. Rev. A 12 (1975) 1106.

[10] Singh, H. B., Sharma, A. and Pathak, K. N., Phys. Rev. A 19 (1979) 899.

[11] Coste, J., Nucl. Fusion 5 (1965) 284, 293. 